\title{
A PRODUÇÃO CIENTÍFICA SOBRE SURF: UMA ANÁLISE A PARTIR DAS PUBLICAÇÕES ENTRE 2000- 2011
}

\author{
Vinicius Zeilmann Brasil \\ Universidade do Estado de Santa Catarina, Florianópolis, Santa Catarina, Brasil \\ Valmor Ramos \\ Universidade do Estado de Santa Catarina, Florianópolis, Santa Catarina, Brasil \\ Ciro Goda \\ Universidade do Estado de Santa Catarina, Florianópolis, Santa Catarina, Brasil
}

\begin{abstract}
Resumo
Este artigo teve como objetivo realizar uma análise da produção científica sobre surf publicada entre janeiro de 2000 e dezembro de 2011. Realizou-se a busca dos artigos nas bases de dados Google Acadêmico, Scielo, Science Direct, Sport Discus e ISI (Web of Science). Selecionaram-se 150 manuscritos que foram analisados e classificados em 5 categorias: Sócio-Antropológica (58), Qualidade de Vida e Saúde (38), Desempenho Esportivo (36), Administrativa (11) e Pedagógica (7). Os resultados indicam que a produção científica sobre surf tem aumentado nos últimos anos e suas iniciativas tem-se originado de diversas áreas do conhecimento. Dentre as temáticas mais estudadas verificaram-se aquelas ligadas ao espaço de prática do surf e as lesões oriundas da prática.
\end{abstract}

Palavras-chave: Esportes. Educação Física e Treinamento. Ensino Superior.

\section{Introdução}

emelhante a outras áreas, a consolidação da Educação Física enquanto campo de intervenção e de produção de conhecimento parece depender da quantidade e da qualidade dessa produção intelectual e também da sua adequada divulgação. Habitualmente estas divulgações são realizadas através de livros, teses, dissertações, artigos, etc (MOLINA NETO et al., 2006).

As configurações atuais dos meios de divulgação das informações, nomeadamente, a Rede Mundial de Computadores (Internet), bem como o estimulo à pesquisa e pós-graduação, tem servido como propulsores da produção de conhecimento em Educação Física, gerando um grande desafio no que diz respeito à possibilidade de acompanhamento e de atualização sistemática dos conhecimentos produzidos e, na velocidade com que são divulgados (SILVERMAN; SKONIE, 1997). 
Nesta perspectiva Gilbert e Trudel (2004), sugerem que o acesso a uma forma resumida e sistematizada destes conhecimentos pode auxiliar os profissionais e pesquisadores no acompanhamento da produção científica sobre determinado tema. De fato, as iniciativas em mapear e avaliar a produção de conhecimento no âmbito da Educação Física, ainda que de forma pouco expressiva, tem se tornado recorrente. Estes trabalhos foram inicialmente representados por Faria Júnior (1992) e Gaya (1994), e mais recentemente por Ludorf (2002), Molina Neto et al. (2006) e Rosa e Leta (2010).

Os estudos desta natureza parecem ser típicos de campos acadêmicos em estado de crescente consolidação ou já consolidados, tendo se configurado muitas vezes como uma necessidade para ampliar a reflexão e definir os direcionamentos do seu próprio desenvolvimento, identificando tendências investigativas no âmbito de temáticas, procedimentos metodológicos e abordagem teórica (MOLINA NETO et al., 2006).

Independentemente da área, estas investigações possuem uma característica em comum, nomeadamente o fato de utilizarem como fonte de dados à literatura disponível sobre um determinado tema, já publicada. Segundo Cordeiro et al. (2007), as pesquisas com esta orientação têm recebido duas definições distintas. A primeira refere-se ao modo mais tradicional denominada de revisão narrativa, em que a busca da literatura não se dá de maneira rígida e específica, ou seja, a seleção dos estudos é aleatória, pessoal, arbitrária e independe de diretrizes pré-estabelecidas. Por outro lado, as revisões que utilizam um método sistemático e mais rigoroso têm sido denominadas de Revisão Sistemática.

Embora este tipo de revisão tenha emergido das Ciências Biológicas, outras áreas já tem se apropriado deste modelo, a exemplo da Educação Física. Principalmente por que auxilia na construção de uma representação simplificada do contexto analisado, da distribuição cronológica da produção, do panorama das temáticas mais estudadas, das orientações metodológicas adotadas e dos rumos investigativos pouco explorados (CORDEIRO et al. 2007).

Relativamente às tendências para as práticas esportivas na natureza, o surf tem se destacado como uma possibilidade importante de intervenção para o profissional de Educação Física, em especial o bacharel (MOREIRA, 2009). Nesta perspectiva, a elaboração de um guia de fontes e informações poderá contribuir para a formação e 
qualificação profissional, ampliando o reconhecimento do surf para o âmbito da intervenção do professor e da investigação científica esportiva.

Neste sentido, o objetivo deste estudo foi analisar a produção científica sobre o surf no período de janeiro de 2000 a dezembro 2011. Especificamente, os objetivos são: elaborar um panorama do volume de publicações; verificar em quais periódicos estão sendo divulgados estes conhecimentos; e identificar as tendências temáticas das investigações sobre esta modalidade.

\section{Método}

Realizou-se uma análise bibliográfica sobre surf, que consiste no levantamento e utilização de materiais escritos já publicados (ALVES-MAZZOTTI; GEWANDSZNAJDER, 2004).

A análise da bibliografia ocorreu em duas etapas: A primeira, de caráter quantitativo, consistiu na identificação e seleção dos estudos sobre surf, utilizando-se procedimentos de estudos de revisão sistemática. Este procedimento possibilitou a síntese, apresentação e identificação de tendências temáticas de investigação

Os procedimentos de revisão sistemática foram os seguintes: a) Definição dos critérios de inclusão, ou seja, foram considerados artigos publicados em periódicos científicos sobre surf (estudos empíricos, de revisão, relato de experiência, ou ainda ensaio teórico), entre janeiro de 2000 e dezembro de 2011, nos idiomas português, espanhol ou inglês, com pelo menos o resumo disponível; b) Seleção das bases de dados eletrônicos ISI (Web of Science), Sport Discus, Science Direct, Scielo e Google Acadêmico; c) definição dos descritores para a busca dos estudos "Surfe", "Surf", "Surfing", "Surfers", "Sport" e "Esporte" d) Busca e seleção dos manuscritos, utilizando-se a combinação dos descritores pelos operadores lógicos "and", "or" e "not", tendo como marcador o "título", "resumo" ou "qualquer lugar do artigo".

Com base nos critérios de seleção e ainda, analisando os títulos, palavras-chave e resumos, principalmente indicadores encontrados no objetivo e população do estudo, foram selecionados 150 artigos. Sendo disponibilizados 108 artigos na sua forma completa e 42 na forma de resumo. 
A segunda etapa da análise da produção científica sobre surf foi realizada com base na proposta de Faria Júnior (1992) e Gaya (1994), para a sistematização dos enfoques temáticos de pesquisa em Educação Física (Systematisation for Research Approaches in Physical Education - SRAPE). Para este estudo, portanto, as pesquisas

foram classificadas em cinco categorias: Sócio-Antropológica; Qualidade de Vida e Saúde; Desempenho Esportivo; Administrativa e Pedagógica. Para a classificação dos artigos, utilizaram como unidades de análise, termos encontrados no título, objetivo, problema, amostra ou sujeitos do estudo.

A fim de garantir níveis aceitáveis de fiabilidade, relativamente ao processo de classificação dos artigos, utilizou-se o seguinte procedimento: Primeiramente, os investigadores reuniram-se e classificaram todos os 150 artigos. Este procedimento se repetiu em mais dois momentos posteriores, em intervalo de tempo que, acredita-se, não interferiu nas avaliações anteriores.

\section{Distribuição das publicações sobre surf}

Relativo ao ano de publicação dos artigos interpretou-se oportuno apresentá-los distribuídos ano a ano e destacados em dois grupos: Nacionais (estudos publicados em periódicos nacionais) e Internacionais (estudos publicados em periódicos estrangeiros), possibilitando a comparação entre diferentes contextos (Gráfico 1).

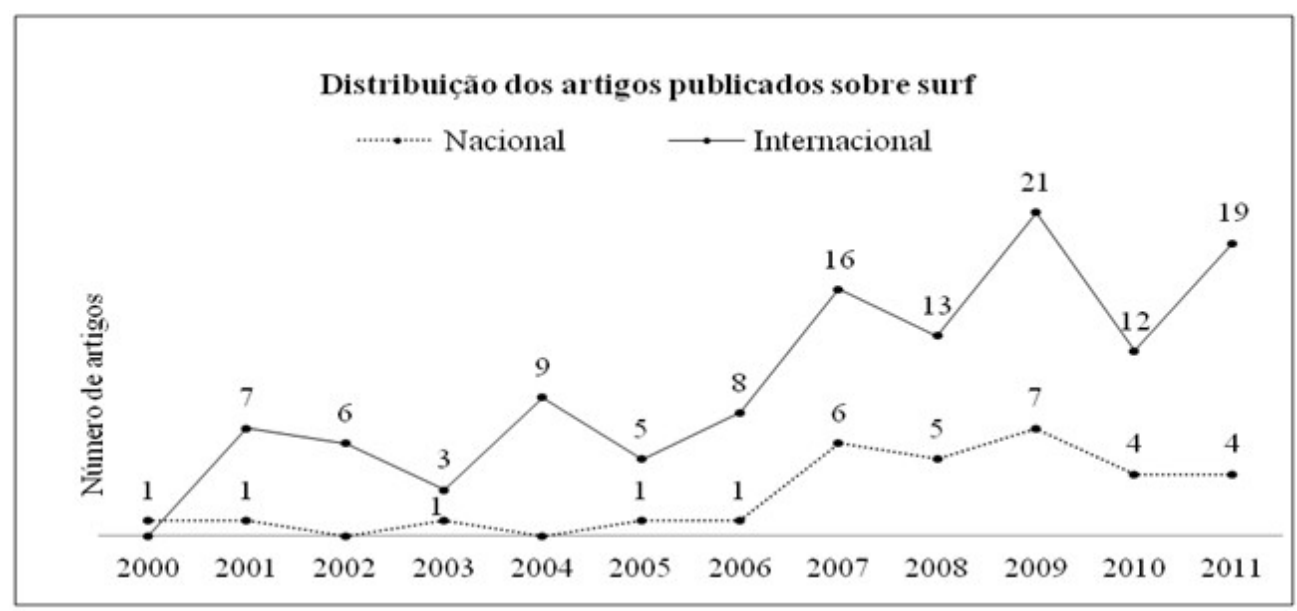

Gráfico 1: Distribuição dos estudos sobre surf publicados no período de Janeiro 2000 à Dezembro 2011. 
A distribuição cronológica dos estudos selecionados (150 artigos) evidencia um crescimento contínuo nas publicações, com destaque para os últimos cinco anos onde a quantidade de artigos publicados correspondeu a $71 \%$ (107 artigos).

A maior parte das pesquisas sobre surf têm sido divulgada no exterior (79\%), destacadamente nos Estados Unidos da América (46), Reino Unido (24), Austrália (16) e Argentina (11); e em menores proporções no Brasil (31 artigos/21\%).

É importante frisar que apesar da produção científica sobre surf se tornar mais expressiva somente nos últimos anos, há indícios de que as primeiras iniciativas puderam ser observadas a partir da década de 80, destacadamente na Austrália e Estados Unidos da América (MOREIRA, 2009).

Com relação aos periódicos científicos (102) em que foram publicados os artigos sobre surf ficou evidente a contribuição de algumas áreas das ciências aplicadas ao esporte (Sociologia, História, Medicina, Economia, Geografia, etc).

No contexto internacional destacou-se o Journal of Coastal Research (11 artigos) considerado um dos principais periódicos para estudos relacionados aos ambientes costeiros (de água doce, salobra ou salgada) e processos de utilização, proteção e gestão desses recursos. Também filiado a Coastal Education \& Research Foundation-CERF.

Por sua vez, a revista Lecturas, Educación Física y Deportes (11 artigos) divulga trabalhos sobre temas da Educação Física, esportes, atividade física de aventura na natureza, treinamento esportivo, recreação e lazer, etc.

Destaca-se também o Journal of Science and Medicine in Sport (8 artigos), que publica estudos sobre lesões esportivas (epidemiologia e prevenção), reabilitação e gestão de lesões, nutrição esportiva e atividade física e saúde, na área da Educação Física e esportes, Medicina, Fisioterapia e Terapia Ocupacional.

Dentre os periódicos que tem acolhido boa parte da produção nacional sobre Educação Física e Esporte, as revistas que tem publicado mais sobre o surf são: revista FIEP BULLETIN (3 artigos); Revista Brasileira de Ciência e Movimento (3 artigos); Revista Brasileira de Medicina do Esporte (3 artigos); Revista Brasileira de Educação Física e Esporte (2 artigos); e Movimento: Revista da Escola de Educação Física (2 artigos). 


\section{Enfoques temáticos dos estudos sobre surf}

Referente à classificação dos estudos nas categorias gerais de pesquisa em Educação Física (Sócio-Antropológica, Desempenho Esportivo, Qualidade de Vida e Saúde, Administrativa e Pedagógica), adaptadas das propostas de Faria Júnior (1992) e Gaya (1994), a Tabela 1 apresenta a classificação dos artigos (representados pelos números de 1 a 150) em categorias, subcategorias e temáticas de investigação.

Tabela 1: Classificação dos estudos sobre surf.

\begin{tabular}{|c|c|c|}
\hline Ctgs. & Subcategorias & Temáticas de investigação (númer o r efer ente a lista dos artigos) \\
\hline \multirow{5}{*}{$\begin{array}{c}3 \\
0 \\
0 \\
0 \\
0 \\
0 \\
0 \\
0 \\
0 \\
0 \\
0 \\
0 \\
0 \\
0 \\
0\end{array}$} & $\begin{array}{l}\text { Espaços de } \\
\text { prática do surf }\end{array}$ & $\begin{array}{l}\text { Caracter isticas da formação e arr ebentação das ondas }(1,2,3,4,5,6,7 \text { e } 8) \text {; } \\
\text { Impacto ambiental e gestão dos espaços de prática }(9,10,11,12,13 \text { e 14); } \\
\text { Construção de espaços para a prática do surf }(15,16,17,18 \text { e 19). }\end{array}$ \\
\hline & $\begin{array}{l}\text { História e } \\
\text { popularização }\end{array}$ & $\begin{array}{l}\text { História do surf nas mídias }(20,21,22,23,24,25,26,27 \text { e } 28) \text {; } \\
\text { História do surf em diferentes paises }(29,30,31,32,33 \text { e } 34) \text {. }\end{array}$ \\
\hline & $\begin{array}{l}\text { Comportamentos } \\
\text { e subcultura surf }\end{array}$ & $\begin{array}{l}\text { Comportamentos de surfistas dos gêner os masculino e feminino }(35,36,37,38 \text {, } \\
39,40,41,42 \text { e } 43) ; \\
\text { Influência da midia na configuração dos comportamentos ( } 44 \text { e } 45) ;\end{array}$ \\
\hline & $\begin{array}{l}\text { Valores e a desão } \\
\text { à prática }\end{array}$ & $\begin{array}{l}\text { Motivos de adesão à prática surf }(46,47,48 \text { e } 49) \text {; } \\
\text { Sentidos evalor es do surf }(50,51 \text { e } 52)\end{array}$ \\
\hline & $\begin{array}{l}\text { Interações na } \\
\text { prática }\end{array}$ & $\begin{array}{l}\text { Comp artilhamento dos espaços de prática (localismo) }(53,54,55 \text { e } 56) \text {; } \\
\text { Interações entre o surfista e a natur eza }(57 \text { e } 58)\end{array}$ \\
\hline \multirow{2}{*}{ 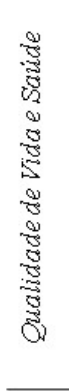 } & Lesões no surf & $\begin{array}{l}\text { Tipos e ocorrência de lesões no surf }(59,60,61,62,63,64,65,66,67,68,69 \text {, } \\
70,71,72,73,74,75,76,77,78 \text { e } 79) ; \\
\text { Lesóes na coluna de surfistas }(80,81,82,83 \text { e } 84) ; \\
\text { Enfermidades gastrointestinais em praticantes de surf( }(85 \text { e 86); } \\
\text { Lesóes na pele ( } 87) ; \\
\text { Lesóes ocular es ( } 88) ; \\
\text { Percepção de risco em relação às lesões na cabeça (89); }\end{array}$ \\
\hline & $\begin{array}{l}\text { Condições de } \\
\text { saúde }\end{array}$ & $\begin{array}{l}\text { Incidência de afogamentos e tempo de exposição ao mar (90 e 91); } \\
\text { Educação em saúde e enfermagem para atletas de surf(92 e 93); } \\
\text { Promoção e manutenção da saúde mental ( } 94 \text { e } 95 \text { ); } \\
\text { Condições de saúde no trabalho de fabricantes de pranchas (96); }\end{array}$ \\
\hline \multirow{5}{*}{ 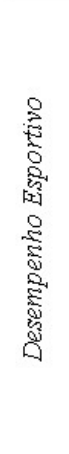 } & $\begin{array}{l}\text { Variáveis } \\
\text { fisiológicas }\end{array}$ & $\begin{array}{l}\text { Comportamento da freqüência car díaca no surf }(97,98,99,100,101 \text { e } 102) \text {; } \\
\text { Demanda energética durante a prática do surf }(103,104,105,106 \text { e 107); } \\
\text { Capacidades físicas utilizadas na prática do surf }(108 \text { e 109). }\end{array}$ \\
\hline & $\begin{array}{l}\text { Treinamento } \\
\text { esportivo }\end{array}$ & $\begin{array}{l}\text { Caracteristicas do tr einamento de surfistas }(110,111,112,113,114 \text { e } 115) \text {; } \\
\text { Treinamento postural e de força em surfistas }(116,117 \text { e } 118) \text {. }\end{array}$ \\
\hline & $\begin{array}{l}\text { Variáveis } \\
\text { psicológicas }\end{array}$ & $\begin{array}{l}\text { Traços de personalida de e desempenho de surfistas }(119,120 \text { e } 121) \text {; } \\
\text { Niveis de ansieda de em competidor es de surf }(122,123 \text { e } 124) \\
\text { Tempo de reação de atletas de surf }(125)\end{array}$ \\
\hline & Cinésiologia & $\begin{array}{l}\text { Comp ortamento muscular de surfistas (126 e } 127) \text {; } \\
\text { Controle postural e força muscular isocinética de atletas de surf(128 e 129). }\end{array}$ \\
\hline & Biomecânica & Tempo de movimento realiza do durante a prática $(130,131$ e 132$)$ \\
\hline
\end{tabular}




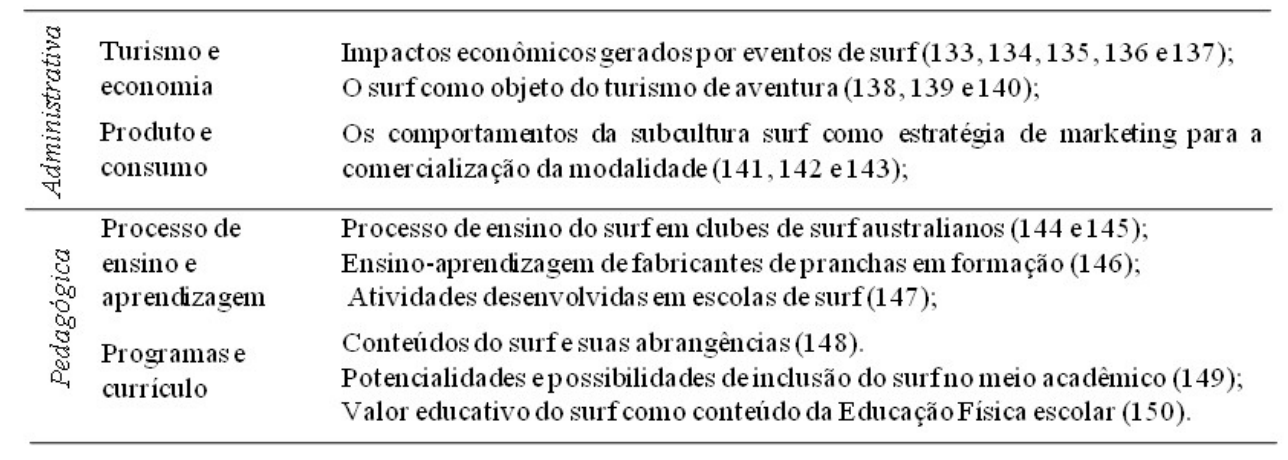

A maior parte dos estudos incidiu sobre as categorias Sócio-Antropológica (58 artigos), Qualidade de Vida e Saúde (38 artigos) e Desempenho Esportivo (36 artigos). Em contrapartida, os artigos classificados nas categorias Administrativa (11 artigos) e Pedagógica (7 artigos) corresponderam a uma parte menos expressiva das publicações selecionadas.

A respeito dos estudos classificados na categoria Sócio-Antropológica os espaços de prática (praia e o mar), nomeadamente as características das ondas, a exploração e conservação das zonas costeiras pelos praticantes e a construção de espaços artificiais para a prática do surf tem recebido maior atenção por parte dos investigadores.

A interpretação dos comportamentos e atitudes de surfistas de ambos os gêneros tem recebido igual atenção. Em termos gerais, estes estudos mostram que o surf tem sido considerado pelos seus praticantes um "modo de levar a vida", de se expressar, e capaz de definir comportamentos em nível físico, psicológico e espiritual, tendo como exemplo os escritos de Evers (2009).

Outras questões importantes debatidas na literatura recaem sobre o que tem levado as pessoas a envolverem-se com o surf. A busca pelo ambiente natural "praia e mar" associado às sensações de lazer, o "descompromisso" com rendimento técnico, o culto ao corpo, um estilo de vida saudável, e ainda o sentimento de aventura, o risco e a vertigem proporcionadas pela prática, tem sido os principais motivos para a experimentação e adesão à prática do surf, dos quais destaca-se o trabalho de Treadwell, Kremer e Payne (2007).

As interações estabelecidas no contexto de prática do surf também têm sido exploradas sob dois enfoques: o primeiro diz respeito ao "localismo" como uma representação da identidade predominante nas relações sociais entre os surfistas, o qual estabelece os limites e os 
conflitos das interações entre os praticantes. De acordo com Gomes et al., (2009), o "localismo" tem sido responsável por práticas protecionistas em relação às praias e ao grupo, estimulando, algumas vezes, atitudes discriminatórias e violentas.

No segundo direcionamento, as pesquisas retratam que a relação do surfista com o espaço da prática (praia o mar) representa a liberdade, a construção de elos com o natural, o culto ao lúdico, etc. Por outro lado, como sugerem Bandeira e Rúbio (2011), a sintonia do surfista com o mar e certo domínio sobre ele, significa a cumplicidade do homem com a natureza, o privilégio de tornar-se surfista, ou seja, possibilidades de corpos corajosos e hábeis.

Referente à categoria Qualidade de Vida e Saúde os debates têm incidido sobre a epidemiologia das lesões no surf e as condições de saúde dos surfistas. Segundo Nathanson et al., (2007), as lesões traumáticas são mais frequentes, seguidas de entorses, distensões e fraturas. Vale frisar que as lesões geradas pelo o esforço repetitivo também são comuns entre os praticantes, sobretudo nas regiões lombares, dos ombros e joelhos.

Sobre a qualidade de vida, os estudos confirmam a imagem que o surfista representa de individuo com hábitos saudáveis e boa qualidade de vida, verificado no estudo de Fleishmann et al. (2011) sobre os benefícios físico e mental proporcionados pela prática do surf. Por outro lado, Norisue et al. (2009) indica os riscos e as possíveis enfermidades desencadeadas pela exposição demasiada ao ambiente de prática.

Os estudos sobre a categoria Desempenho Esportivo estão direcionados, em maior parte, aos aspectos fisiológicos, tanto no âmbito da prática competitiva como recreativa. De acordo com Brasil et al. (2001) a análise do comportamento da freqüência cardíaca de praticantes de surf recreacional, sugere que a intensidade de uma sessão de surf pode ser considerada moderada, possuindo um aumento na média na freqüência no momento em que o surfista está na onda.

No âmbito da prática competitiva, o destacado estudo de MendezVillanueva et al. (2005), mostram que o consumo de oxigênio obtido em competidores é sistematicamente superior aos valores verificados em surfistas recreacionais. De modo geral, os estudos sobre a modalidade têm mostrado que o desempenho no surf é decorrente de diferentes capacidades físicas e adaptações fisiológicas, com nível de exigência variado durante toda a sessão de prática. 
A identificação dos padrões e o tempo de movimentos dos surfistas também tem sido tema de pesquisa. Tem se verificado que a maior parte do tempo de uma sessão de surf esta destinada a execução dos fundamentos da remada e o "sentar na prancha", respectivamente, utilizados para pegar a onda e retornar ao "outside" e, aguardar a chegada das ondas, conforme estudos de Mendez-Villanueva, Bishop e Hamer (2006).Igualmente, as variáveis psicológicas, em particular, os traços de personalidade e a ansiedade são tratadas nos estudos sobre o desempenho de atletas de surf. De modo geral, há indícios de que os comportamentos destas variáveis estão relacionados à experiência de prática dos atletas, bem como aos elementos que os motivam para a prática, verificado no estudo de García e Romero (2009).

Referente ao treinamento de atletas de surf, o trabalhos de Mendez-Villanueva, Mujika e Bishop (2010), alertam para a importância da periodização e do treinamento específico para o surf, ainda que a rotina de viagens e competições dos atletas e a instabilidade do local de prática se configurem como desafios para esta sistematização.

As discussões mais recorrentes sobre a categoria Administrativa tem se referido ao surf como produto de consumo do turismo de aventura. Esta modalidade esportiva já tem sido considerada um fenômeno prevalente e crescente no mercado mundial, verificado por Buckley (2002).

No âmbito do turismo as atividades estão centradas na busca por regiões e locais ainda não explorados para a prática do surf, as quais têm estabelecido alguns padrões de consumidores, destacadamente aqueles que buscam condições específicas de ondas, locais de longo tempo de permanência e praias adequadas para surfistas de diferentes níveis de prática (DONILKAR; FLUKER, 2003). Além disso, os comportamentos da subcultura surf, principalmente a moda surf, a linguagem, a música, já são utilizados como estratégia de marketing para a comercialização e a massificação da prática da modalidade, referenciado por Moutinho, Dionísio e Leal (2007).

Dentre as pesquisas classificadas na categoria Pedagógica uma parte dela incide sobre a descrição e interpretação da aprendizagem e da formação da identidade de crianças e jovens em clubes de surf, com aporte nos conceitos de comunidade da prática e aprendizagem situada de Jeane Lave e Etienne Wenger, o qual destaca a importância

1-Zona de surf antes da linha de arrebentação das ondas, sentido mar costa. 
do envolvimento em um determinado grupo social para a aprendizagem e incorporação de atitudes, valores e habilidades de uma comunidade específica (LIGHT; NASH, 2006).

A outra parte das investigações destaca as possibilidades do surf como prática a ser incorporada pelos currículos de cursos de ensino superior, sugerindo a introdução de elementos curriculares não convencionais, especificamente no âmbito da formação profissional em Educação Física (VAGHETTI; PARDO, 2007). Em nível de educação básica, tem se verificado a importância do surf, como conteúdo da Educação Física, no desenvolvimento de valores educacionais pautados no condicionamento físico, habilidades físicas e atitudes relacionadas à utilização e conservação do meio ambiente, conforme o estudo de Vera (2008).

Contudo, relativamente ao predomínio dos estudos de ordem biológica no âmbito da produção sobre surf, em parte, corrobora com os achados de Faria Júnior (1992) e Gaya (1994), os quais já destacavam que no decorrer do desenvolvimento da Educação Física, ainda que a produção científica tenha transitado por diversos campos do conhecimento, sua produção esteve tradicionalmente ligada as Ciências Naturais, sobretudo às áreas médica e biológica. Esta tendência tem se refletido ainda nos tempos atuais, como mostram Rosa e Leta (2010) em seu estudo sobre as tendências atuais da pesquisa brasileira em Educação Física, verificando uma maior valorização dos conhecimentos advindos das Ciências Biológicas.

Acredita-se também que o aumento nos estudos biológicos sobre surf, particularmente com enfoque no Desempenho Esportivo, pode ter alguma relação com a incorporação dos valores das sociedades industriais às práticas esportivas atuais, marcadas pelo rendimento, hierarquização, organização burocrática e publicidade; vindo a se consolidar como uma prática esportiva profissional nos anos de 1990.

De acordo com Mendez-Villanueva, Bishop e Hamer (2006) a análise dos fatores de rendimento impostas pela prática competitiva no surf tem seguido orientações semelhantes às modalidades tradicionais, como o futebol, basquetebol e o hóquei.

Para além das iniciativas voltadas ao rendimento esportivo, os estudos com abordagem Biológica têm incidido também sobre o enfoque Qualidade de vida e saúde. O que se percebe é que estes estudos têm surgido como uma necessidade, em função do aumento no volume de adeptos nas últimas décadas. Apesar da prática do surf estar as- 
sociada à saúde, os aspectos da segurança dos praticantes têm chamado à atenção dos investigadores.

Relativamente ao volume das pesquisas com enfoque Sócio-antropológico, é nítida a contribuição de investigadores da antropologia, sociologia, história, geografia, oceanografia. Acredita-se que a contribuição destas áreas decorre da forma com esta modalidade foi sendo incorporada e popularizada ao longo do tempo.

A prática do surf esteve ligada primeiramente a algumas idéias de contestação de modos padronizados de agir e de pensar das pessoas nas sociedades urbanas e tecnocráticas, das décadas de 1960 e 1970 (BUTTS, 2004). A forma de contestação se dava principalmente através da adoção de valores e modos de agir contrários aos adotados nestas sociedades, nomeadamente, valores ligados à busca do prazer, liberdade, união com a natureza, amizade, humildade, lealdade e demais valores que pudessem estabelecer inclusive uma nova cultura ou uma "contracultura". Assim, cria-se um novo modo de se vestir, diferente e despojado, com uma linguagem e modo de ser muito particular.

Posteriormente, o surf deixa de ser visto somente como uma prática de formação identitária, passando a ser valorizado em sua face recreativa e de lazer. Torna-se, portanto, uma alternativa de prática destinada ao tempo livre, atraindo as pessoas para as vivências de lazer. O contato direto com a natureza, bem como as emoções e sensações proporcionadas a partir da interação do surfista com o mar e sua prancha, parecem contrapor as exigências do "mundo do desporto tradicional", contribuindo para o aumente de adeptos a essa modalidade esportiva (THORPE, 2006).

Assim, este conjunto de características típicas do surf, ligadas aos aspectos culturais, as interações com o ambiente natural e as sensações advindas desta prática, o aumento do número de praticantes, parecem ser os elementos propulsores da produção científica com este enfoque.

Outra tendência que se configura no panorama da produção científica sobre surf é a pouca incidência dos estudos pedagógicos, em contraste com o predomínio dos estudos sócio-antropológicos (categoria Sócio-Antropológica) e biológicos (categorias Desempenho Esportivo e Qualidade de Vida e Saúde).

Acredita-se que essa pouca incidência deva ser vista com preocupação, haja vista que a popularização do surf como prática de lazer e 
de competição, tem se refletido na ampliação do número de praticantes em vários países, transformando este esporte em um campo importante de intervenção profissional, seja para orientar crianças e jovens em um processo de iniciação esportiva, para orientar atividades de treinamento esportivo, ou ainda, para atender as demandas de lazer.

Esta nova demanda de intervenção, implica igualmente um nível adequado de qualificação e preparação pedagógica para atender às exigências da prática. Por definição, o surf é um esporte de natureza realizado em meio líquido instável, dotado de certa imprevisibilidade e capaz de proporcionar sensações de risco e harmonia entre o praticante e a natureza (FUNOLLET, 1995). Estas características adicionam dificuldades à tarefa do professor, à medida que se exige dele, a elaboração de situações de aprendizagem, que proporcionem níveis seguros de prática e também de preservação das sensações de prazer ao praticante.

De modo geral, a formação de profissionais para intervir no ensino ou na orientação de atividades práticas do surf, tem sido realizada por iniciativas particulares de associações, confederações e institutos específicos da modalidade. Embora estas iniciativas sejam relevantes, ao serem desenvolvidas isoladamente, podem não lograr êxito a médio e longo prazo. De um lado, pela necessidade de instrutores ou professores para contemplar o aumento do número de praticantes e, por outro lado, pelas demandas de ampliação e aprofundamento dos conhecimentos científicos necessários para intervir de forma adequada, por exemplo, no âmbito do esporte competitivo e profissional.

Neste sentido, os cursos de formação inicial em Educação Física podem exercer um papel central na preparação pedagógica de profissionais para atender a esta nova demanda de intervenção esportiva, principalmente na realização de estudos que ampliem os conhecimentos pedagógicos disponíveis sobre o surf.

A expectativa, portanto, é que estas recentes alterações curriculares, que transferem aos cursos bacharelado a responsabilidade de preparar profissionais para atuar neste contexto da cultura corporal e esportivo em particular, associadas a outras formas de estímulo à produção de conhecimento para área de Educação Física possam contribuir para o aumento dos conhecimentos pedagógicos do professor, assim como tem ocorrido nas dimensões mais tradicionais da área. 


\title{
Considerações finais
}

Deve-se destacar primeiramente, que o volume e a qualidade das pesquisas sobre essa modalidade, já permitem identificar um corpo de estudos com características e enfoques de pesquisa definidas. Desse modo, com o propósito de contribuir para este processo, pode-se afirmar que os resultados da analise dos estudos sobre surf indicam que:

Os estudos sobre surf têm sido publicados em uma gama variada de periódicos, destacadamente em revistas científicas da área da Geografia, Oceanografia, Medicina, Sociologia, História e Educação Física.

O aumento na produção científica parece estar mais fortemente, vinculado às demandas da prática e sua trajetória de inserção na sociedade ao longo dos anos. Por outro lado, os incentivos fornecidos aos programas de pós-graduação em diversas áreas do conhecimento, inclusive na Educação Física, também tem contribuído para o aumento das publicações sobre a modalidade.

O estado atual da produção científica sobre surf sugere uma exploração ampla do tema. Apesar da dispersão, as investigações apontam para o predomínio de estudos no âmbito sócio-antropológico (Socioantropológica) e biológicos (Desempenho Esportivo e Qualidade de Vida e Saúde) e, em menor proporção as iniciativas de investigações Pedagógicas (Pedagógica).

A síntese de dados ainda dispersos, pôde gerar resultados que atendem a carência da literatura sobre esta modalidade, possibilitando a elaboração de um guia de estudos e sugerindo novos rumos investigativos.

O surf, enquanto pratica esportiva, já se configura como um campo de intervenção e estudo para o profissional de Educação Física, o que gera uma expectativa a respeito do modo como as entidades esportivas, entidades profissionais e as faculdades de Educação Física, contribuirão na formação do profissional para atuar neste contexto.

\section{THE SCIENTIFIC PRODUCTION ABOUT SURF: AN ANALYSIS FROM PUBLICATIONS BETWEEN 2000-2011}

\begin{abstract}
This article aims to perform an analysis of scientific literature on surfing published between January 2000 and December of 2011. The search of articles was conducted on databases Google Scholar, Scielo, Science Direct, and Sport Discus (ISI
\end{abstract}


Web of Science). 150 manuscripts were selected, analyzed and classified into five categories: Socio-Anthropological (58), Quality of Life and Health (38) Sports Performance (36), Administrative (11) and Educational (7). The results indicate that the scientific production about surfing has increased in recent years and its initiatives have been originated from different areas of knowledge. Among the topics most studied there were those related to the location of surfing and injuries arising from the practice.

Keywords:Sports. Physical Education and Training. Higher Education.

\section{LA PRODUCCIÓN CIENTÍFICA SOBRE EL SURF: UN ANÁLISIS DE LAS PUBLICACIONES DE ENTRE 2000-2011}

\section{Resumen}

Este artículo tiene como objetivo realizar un análisis de la literatura científica sobre el surf publicados entre enero de 2000 y diciembre de 2011. Se realizó una búsqueda de artículos en las bases de datos Google Scholar, Scielo, Science Direct, Sport Discus y ISI (Web of Science). Fueron seleccionado 150 manuscritos después analizadas y clasificadas en cinco categorías: Socio-Antropológica (58), Calidad de Vida y Salud (38) Rendimiento Deportivo (36), Administrativo (11) y Pedagógico (7). Los resultados indican que la producción científica sobre el surf se ha incrementado en los últimos años y sus iniciativas ha sido su origen en diferentes áreas del conocimiento. Entre los temas más estudiados fueron los relacionados con el espacio de práctica y lesiones derivadas de la práctica.

Palabras clave:Deportes. Educación y Entrenamiento Físico. Educación Superior.

\section{Referências}

ALVES-MAZZOTTI, A. J.; GEWANDSZNAJDER, F. O método nas ciências naturais e sociais: pesquisa quantitativa e qualitativa. São Paulo: Pioneira/Thompson Learning, 2004.

BANDEIRA, M. M.; RÚBIO, K. "Do outside": corpo, natureza, medo e gênero no surfe universitário paulistano. Revista Brasileira de Educação Física e Esporte, São Paulo, v. 25, n. 1, p. 97-110, jan./mar., 2011.

BRASIL, F. K. et al. Freqüência cardíaca e tempo de movimento durante o surfe recreacional - estudo piloto. Revista Brasileira de Ciências do Movimento, Brasília, v. 9, n. 4, p. 65-75, out., 2001.

BUCKLEY, R. Surf tourism and sustainable development in Indo-Pacific Islands. Journal of Sustainable Tourism, v. 10, n. 5, p. 405-424, 2002. 
BUTTS, S. L. "Good to the last drop": understanding surfers motivations. Sociology of Sport, v. 32, n. 2, p. 229-243, 2004.

CORDEIRO, A. M. et al. Revisão sistemática: uma revisão narrativa. Revista do Colégio Brasileiro de Cirurgiões, Rio de Janeiro, v. 34, n. 6, p. 428-431, nov./dez., 2007.

DONILKAR, S.; FLUKER, M. Bihavioural market segments among surf tourists: investigating past destination choice. Journal of Tourism, v. 8, n. 3, p. 186-196, 2003.

EVERS, C. 'The Point': surfing, geography and a sensual life of men and masculinity on the Gold Coast, Australia. Social and Cultural Geography, v. 10, n. 8, p. 893-908, 2009.

FARIA JÚNIOR, A. G. Pesquisa em Educação Física: enfoques e paradigmas. In: FARIA JÚNIOR, A. G.; FARINATTI, P. T. Pesquisa e produção de conhecimento em educação física. Rio de Janeiro: Ao Livro Técnico, 1992, p. 59-68.

FLEISCHMANN, D. M. D. et al. Surf medicine: surfing as a means of therapy for combat - related polytrauma. Journal of Prosthetics and Orthotics, v. 23, n. 1, p. 27-38, 2011.

FUNOLLET, F. Propuesta de clasificación de las actividades deportivas em el médio natural. Apunts: Educación Física y Deportes, Barcelona, v. 41, p. 124-129, jul./set., 1995.

GARCÍA, F. G.; ROMERO, D. N. Autoestima y competitividad em uma selección juvenil peruana de surf. Revista Iberoamericana de Psicología del Ejercicio y del Deporte, v. 4, n. 2, p. 253-270, 2009.

GAYA, A. C. A. As ciências do desporto nos países de língua portuguesa: uma abordagem epistemológica. 1994. 190 f. Tese (Doutorado em Ciências do Desporto e de Educação Física)-Faculdade de Ciências do Desporto e de Educação Física, Universidade do Porto, Porto, 1994.

GILBERT, W. D.; TRUDEL, P. Role of the coach: how model youth team sport coaches frame their roles. The Sport Psychologist, v. 18, p. 21-43, 2004. 
GOMES, M. A. et al. Identidade coletiva dos surfistas de Florianópolis e o fenômeno do localismo. Revista de Psicologia, Florianópolis, n. 1, p. 85-102, 2009.

LIGHT, R.; NASH, M. Learning and identity in overlapping communities of practice: surf club, school and sports clubs. The Australian Educational Researcher, v. 33, n. 1, p. 75-94, 2006.

LUDORF, S. M. A. Panorama da pesquisa em Educação Física da década de 90: análise dos resumos de dissertações e teses. Revista da Educação Física/UEM, Maringá, v. 13, n. 2, p. 19-25, jul./dez., 2002.

MENDEZ-VILLANUEVA, A. et al. Upper body aerobic fitness comparison between two groups of competitive surfboard riders. Journal of Science and Medicine in Sport, v. 8, n. 1, p. 43-51, mar., 2005.

MENDEZ-VILLANUEVA, A.; BISHOP, D.; HAMER, P. Activity profile of world-class Professional surfers during competition: a case study. Journal of Strength and Conditioning Research, Champaign, v. 20, n. 3, p. 477-482, ago., 2006.

MENDEZ-VILLANUEVA, A.; MUJIKA, I.; BISHOP, D. Variability of competitive performance assessment of elite surfboards riders. Journal of Strength and Conditioning Research, Champaign, v. 24, n. 1, p. 135-139, jan., 2010.

MOLINA NETO, V. et al. Reflexões sobre a produção do conhecimento em Educação Física e Ciências do Esporte. Revista Brasileira de Ciências do Esporte, Campinas, v. 28, n. 1, p. 145-165, set., 2006.

MOREIRA, M. Surf: Da ciência a prática. 1. ed. Lisboa: FMH, 2009.

MOUTINHO, L.; DIONÍSIO, P.; LEAL, C. Surf tribal behavior: as sports marketing application. Marketing Intelligence and Planning, v. 25, n. 7 , p. $668-690,2007$.

NATHANSON, A. et al. Competitive surfing injuries: a prospective study of surfing-related injuries among contest surfers. American Journal of Sports Medicine, v. 35, n. 1, p. 113-117, 2007. 
NORISUE, Y. et al. Surfing as a risk factor for gastro esophageal reflux disease. Clinical Journal of Sport Medicine, Nova York, v. 19, n. 5, p. 388-393, set., 2009.

ROSA, S.; LETA, J. Tendências atuais da pesquisa brasileira em Educação Física parte 1: uma análise a partir dos periódicos nacionais. Revista Brasileira de Educação Física e Esporte, São Paulo, v. 24, n. 1, p. 121-134, jan./mar., 2010.

SILVERMAN, S.; SKONIE, R. Research on teaching in Physical Education: an analysis of published research. Journal of Teaching in Physical Education, v. 16, p. 300-311, 1997.

THORPE, H. Beyond "decorative sociology": contextualizing female surf, skate and snowboarding. Sociology of Sport Journal, Champaign, v. 23, n. 3, p. 205-228, set., 2006.

TREADWELL, J.; KREMER, P.; PAYNE, W. The determinants and motives for young people to participate in surfing. Journal of Science and Medicine in Sport, Belconnen, v. 10, n. 6, p. 68-68, 2007.

VAGHETTI, C. A. O.; PARDO, E. R. Um esporte não convencional no mundo acadêmico: singularidades histórico-culturais e possibilidades de inclusão do ensino do surfe na universidade. Fiep Bulletin, v. 78, 2007.

VERA, U. A. Los deportes acuáticos: el surf en el área de Educación Física. Lecturas, Educación Física y Deportes, Buenos Aires, v. 13, n. 126, nov., 2008. Não paginado.

Recebido em: 25/07/2012

Revisado em: 17/11/2012

Aprovado em: 22/01/2013

Endereço para correspondência

d2vr@hotmail.com

Valmor Ramos

Universidade do Estado de Santa Catarina,

Centro de Educação Física e Desportos.

Rua Pascoal Simone, 358

Coqueiros

88080-350 - Florianopolis, SC - Brasil 